\title{
Amoebiasis: a rare cause of cardiac tamponade
}

\author{
L N Gomersall, J Currie, R Jeffrey
}

\begin{abstract}
Cardiac tamponade secondary to perforation of a hepatic amoebic abscess developed six years after the patient had visited an area where Entamoeba histolytica is endemic. He was treated with metronidazole and imipenem, emergency percutaneous catheter drainage, and open surgical drainage.
\end{abstract}

(Br Heart f 1994;71:368-369)

In western industrialised societies most cases of invasive amoebiasis occur after travel to tropical regions where infection by Entamoeba histolytica is endemic. The most common non-enteric complication of amoebiasis is hepatic abscess, which can present after a long latent period. Perforation into the chest or abdomen is associated with high morbidity. We report a case complicated by rupture into the pericardial cavity.

\section{Case report}

A 28 year old previously fit white man presented with a six week history of fever, night sweats, pyrexia, and right-sided pleuritic chest pain radiating to his right shoulder. He had

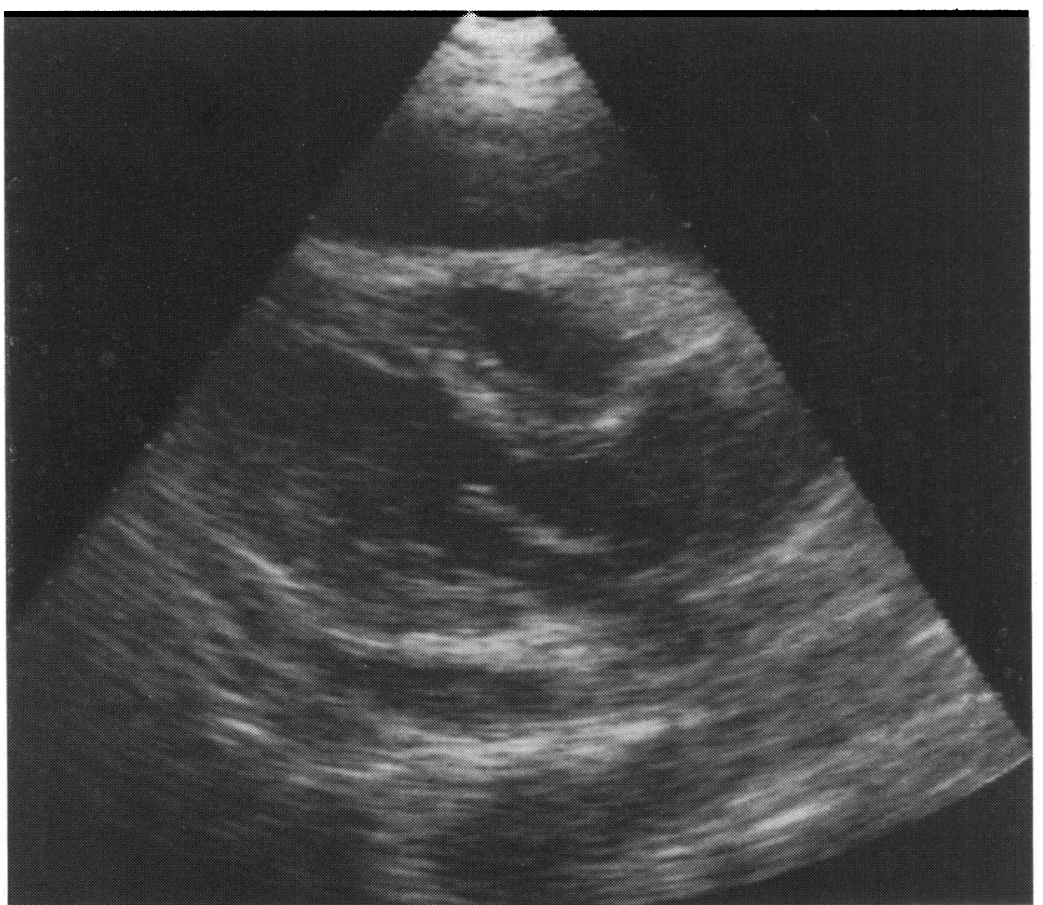

Ultrasound scan, long axis view, showing $2.5 \mathrm{~cm}$ pericardial effusion. lost $9.5 \mathrm{~kg}$ in weight. He was heterosexual with no history of intravenous drug abuse and was a construction worker who had left the army six years before. During military service he was stationed in Kenya but gave no history of tropical disease or episode of ill health.

On examination he was pyrexial $\left(39^{\circ} \mathrm{C}\right)$ with tenderness in the right upper quadrant of the abdomen. Initial investigations showed a mild normochromic, normocytic anaemia with a haemoglobin of $112 \mathrm{~g} / \mathrm{l}$. The white cell count, platelets, and liver function tests were within normal limits. Stool, blood, sputum, and urine cultures were sterile. Serological tests for hepatitis and HIV were negative.

A chest radiograph was normal, but a computed tomography scan of the chest and abdomen showed slight enlargement of the mediastinal nodes, an $8 \mathrm{~cm}$ diameter low density lesion in the left lobe of the liver and $17 \mathrm{~cm}$ splenomegaly. A liver biopsy specimen was obtained under ultrasound control. It showed only fibrous tissue with a small area of normal liver parenchyma. Twenty-four hours later the patient's condition suddenly deteriorated. He complained of right-sided anterior chest pain and was clinically shocked (blood pressure $70 / 42 \mathrm{~mm} \mathrm{Hg}$, pulse 130 ) with both peripheral and central cyanosis.

A repeat ultrasound scan showed a $2.5 \mathrm{~cm}$ diameter pericardial effusion compressing the right ventricular outflow tract and indicating cardiac tamponade (figure). A $5 \cdot 5 \mathrm{~F}$ catheter was inserted parasternally and $400 \mathrm{ml}$ of thin serous brown fluid was drained under pressure. Specimens sent for bacteriological culture were sterile. The possibility of amoebic infection was considered and treatment with metronidazole and imipenem was started. $\mathrm{He}$ rapidly improved, but 24 hours later the small pericardial drain became blocked. A further percutaneous catheter was placed under ultrasound guidance to relieve the impending cardiac tamponade and soon afterwards open pericardial drainage was performed under general anaesthesia. Serous brown fluid drained freely from the pericardial drain and $150 \mathrm{ml}$ of thick pus that resembled anchovy sauce was aspirated percutaneously from the hepatic abscess. We identified a communication through the diaphragm to the hepatic abscess cavity.

Over the next two weeks his condition improved. A fluorescent antibody test for Entamoeba histolytica was positive (titre 1/320) and amoebic enzyme immunoassay was positive at $120 \%$. Treatment with metronidazole was continued for 10 days and a 10 day 
course of diloxanide was given to eradicate any remaining amoebic cysts. At follow up three months later the appearance of the liver had returned to normal; the splenomegaly resolved by six months.

\section{Discussion}

Amoebiasis is readily treatable but a delay in diagnosis can lead to serious complications and even death. Hepatic abscess is the most common non-enteric complication of amoebiasis. Our patient showed some typical features: fever, pain in the right shoulder tip and anterior chest, weight loss, and tender hepatomegaly.

Perforation into the chest or peritoneum is an uncommon but potentially fatal complication of amoebic liver abscess. Thoracic complications are more common; abscesses involving the superior segment of the right lobe of the liver lead to empyema or hepatobronchial fistula. ${ }^{12}$ Although amoebic abscesses are less common in the left lobe of the liver, this site is associated with a higher risk of rupture and more serious sequelae. Mortality from rupture into the pericardium is reported to be $30-60 \% .^{13}$

The tendency for amoebic liver abscess to extend beyond the surface of the liver may aid diagnosis. ${ }^{4}$ Even without frank perforation, reactive serious pleural effusions are common. ${ }^{14}$ Intrapericardial rupture occurred in $<1 \%$ of 501 reported thoracic complications of hepatic amoebic abscess, ${ }^{1}$ and in a series of 148 fatal cases of amoebiasis only two deaths were attributed to cardiac tamponade 5

Medical treatment is standard for intra- hepatic amoebic abscess and is effective in over $90 \%$ of patients. ${ }^{67}$ Recently the role of percutaneous catheter drainage of amoebic abscess has been discussed. ${ }^{6-8}$ There is general agreement that $(a)$ diagnostic aspiration can distinguish between amoebic and pyogenic abscesses when clinical and imaging findings are indistinguishable, and that large (5-10 $\mathrm{cm}$ ) abscesses, particularly in the left lobe of the liver where the risk of perforation is higher, should be drained. The presence of pericardial thickening or effusion is suggestive of pending if not frank pericardial rupture.

In this case percutaneous catheter drainage was a life-saving treatment, but open surgical drainage was required because the catheter used for pericardial drainage was too small to establish continuous drainage.

1 Ibarra-Perez C. Thoracic complications of amoebic abscess of the liver. Report of 501 cases. Chest 1981; 79:672-7.

2 Adams EB, MacLeod IN. Invasive amoebiasis, amoebic liver abscess and its complications. Medicine 1977; 56:325-34.

3 Ken JG, Van Sonnenberg E, Casola G, Christensen R, Polansky AM. Perforated amoebic liver abscesses: successful percutaneous treatment. Radiology 1989;170: cessful

4 Radin R, Ralls P, Colletti PM, Halls JM. CT of amoebic liver abscess. Am $\mathcal{F}$ Roentgenol 1988;150:1297-301.

5 Kean BH, Gilmore HR Jr, Van Stone WW. Fatal amoebiasis: report of 148 fatal cases from the Armed Forces Institutes of Pathology. Ann Intern Med 1956;44: 831-43.

6 Van Sonnenberg E, Mueller PR, Schiffman HR, et al. Intrahepatic amoebic abscesses: indications for and results of percutaneous catheter drainage. Radiology 1985;156:631-5.

7 Ralls PW, Barnes PF, Johnson MB, De Cock KM, Radin $R$, Halls J. Medical treatment of hepatic amoebic abscess: rare need for percutaneous drainage. Radiology 1987;165:805-7.

8 Saraswat DK, Agarwal DK, Baijal SS, et al. Percutaneous catheter drainage of amoebic liver abscess. Clin Radiol 1992;45:187-9. 